\title{
Complex Formation between Gallium(III) and Adriamycin in Aqueous Solutions
}

\author{
Toshihisa Yotsuyanagi, ${ }^{*}$ Naoko Ohta, Norihiro TanaKa, and Ken Ikeda \\ Faculty of Pharmaceutical Sciences, Nagoya City University, Tanabedori 3-1, Mizuho-ku, Nagoya 467, Japan. Received April 26, 1990
}

The complex formation between gallium(III) and adriamycin (ADR) was investigated in $0.1 \mathrm{M} \mathrm{KNO}$ at $25^{\circ} \mathrm{C}$. With excess concentration of $\mathrm{Ga}$ (III) compared to ADR, Ga(III) was found to be coordinated with ADR in the low pH region below about 4.0. The spectrophotometric titration data supported the hypothesis that one proton is released as a result of complexation between Ga(III) and ADR via a $1: 1$ stoichiometry, and a phenolic oxygen on the ADR is involved. The stability constant of the complex was obtained by two different spectrophotometric methods. First, the absorbance development and $\mathrm{pH}$ changes reflecting the release of a proton due to complexation were monitored and the stability constant was estimated using the method of Hildebrand-Reilley. Secondly, ADR was titrated with increasing $\mathrm{Ga}$ (III) at constant pH 3.0 and analyzed by the Benesi-Hildebrand method. We took into consideration gallium hydrolysis in the calculation. The stability constants, $1.24 \times 10^{8}$ and $3.06 \times 10^{8} \mathrm{M}^{-1}$ obtained by the two different methods, showed good agreement within an order-of-magnitude range.

Keywords adriamycin; deprotonation constant; gallium; chelate; metal complex; complex formation

\section{Introduction}

Adriamycin (ADR) is an antitumor antibiotic exhibiting activity against a variety of human cancers. ${ }^{1)}$ However, the presence of dose-limiting toxic side effects has stimulated the development of analogs with the hope of improved efficacy and tolerability without cardiotoxicity.

Complexation of ADR with metal ions has been examined for this purpose. Quelamycin, consisting of three ADR molecules chelated to one $\mathrm{Fe}$ (III) ion, was reported to produce less cardiotoxicity as compared to ADR alone. ${ }^{2)}$ Physicochemical characteristics of Fe(III)-ADR and $\mathrm{Fe}(\mathrm{III})$-daunorubicin complexes have been extensively investigated. $^{3)}$ Complexation with $\mathrm{Cu}(\mathrm{II})$ and $\mathrm{Pd}(\mathrm{III})$ has been also studied. ${ }^{4,5)}$

The radionuclide gallium- 67 has been used extensively as a tumor-imaging reagent. ${ }^{6)}$ Injection of the citrate complex $\mathrm{Ga}(\mathrm{III})$ (citrate) ${ }_{2}$ results in enhanced uptake of the metal into a variety of malignant tumors. ${ }^{7)}$ Similar uptake of gallium complexes with other organic ligands may be expected, as a possible antitumor activity of the Fe(III)-ADR complex is suggested. ${ }^{3)}$ In this report we examined the complex formation between $\mathrm{Ga}$ (III) and ADR, and the stability constant was determined by spectrophotometric titration methods.

\section{Experimental}

Chemicals Purified ADR was kindly provided by Kyowa Hakko Kogyo Co., Tokyo. $\mathrm{Ga}\left(\mathrm{NO}_{3}\right)_{3} \cdot x \mathrm{H}_{2} \mathrm{O}$, ethylenediaminetetraacetic acid (EDTA), and 1-(2-pyridylazo)-2-naphthol (PAN) were purchased from Wako Pure Chemical Industries, Ltd., Osaka. All other chemicals were of reagent. grade.

Apparatuses Absorption spectra were recorded on a Shimadzu UV-260 spectrophotometer (Kyoto). Circular dichroism (CD) spectra were obtained on a Jasco J-600 spectrometer (Tokyo). Spectral changes were expressed in terms of the molar absorptivity for absorption spectra, and as $\Delta \varepsilon=\varepsilon_{\mathbf{L}}-\varepsilon_{\mathbf{R}}$ (molar CD coefficient) where $\varepsilon_{\mathbf{L}}$ and $\varepsilon_{\mathbf{R}}$ are the molar absorptivities for left and right circularly polarized lights. Fluorescence spectra were recorded on a spectrofluorophotometer (RF-520, Shimadzu, Kyoto) at an excitation wavelength of $460 \mathrm{~nm}$. $\mathrm{pH}$ measurements were made with a Horiba $\mathrm{F}-7_{\mathrm{LC}}$ digital $\mathrm{pH}$ meter having a precision of \pm 0.005 $\mathrm{pH}$ unit (Tokyo).

Determinations of $\mathbf{G a}$ (III) and ADR The content of Ga(III) was determined by means of EDTA titrations with PAN as an indicator. ${ }^{8)}$ ADR concentration was determined based on the molar absorptivity at $480 \mathrm{~nm}(\varepsilon, 11500){ }^{9)}$ As anthracycline solutions are generally sensitive to light and oxygen, the stock solution was prepared with water bubbled by nitrogen gas for $10 \mathrm{~min}$ and stored at $-20^{\circ} \mathrm{C}$.

Spectrophotometric Titration of ADR Solution All titrations were carried out in $\mathrm{KNO}_{3}$ (ionic strength 0.1 ) at $25 \pm 0.1^{\circ} \mathrm{C}$. ADR $(9.9 \mu \mathrm{M})$ was titrated with a $\mathrm{KOH}$ solution. Carbonate-free $0.0748 \pm 0.0002 \mathrm{M} \mathrm{KOH}$ was prepared adding barium hydroxide to remove the $\mathrm{CO}_{2}$ from the $\mathrm{KOH}$ solution and exchanging barium for potassium by passing a column of Amberlite IR 120B in the potassium form. ${ }^{10}$ ) The $\mathrm{KOH}$ solution was successively increased to $1400 \mu \mathrm{l}$ by a microsyringe. The mixing of the solution was carried out by gently bubbling nitrogen gas. Once the volume of $\mathrm{KOH}$ solution was added, the $\mathrm{pH}$, absorption spectra and absorptivity at $590 \mathrm{~nm}$ of ADR solution were measured. The decreased concentration of ADR by the addition of a $\mathrm{KOH}$ solution was counterbalanced by changing the full scale of a spectrophotometric recorder. Since ADR decomposes at alkaline $\mathrm{pHs},{ }^{11}$ the spectrum was recorded as quickly as possible after the $\mathrm{pH}$ was raised to the desired value. Titrations were performed in triplicate. Protolytic dissociation constants were obtained for each run and the average values were determined by nonlinear least-squares computer analysis. ${ }^{12,13)}$

Spectral Changes of $\mathrm{ADR}$ with $\mathrm{Ga}\left(\mathrm{NO}_{3}\right)_{3} \quad \operatorname{ADR}\left(40 \mu \mathrm{M}\right.$ in $\left.0.1 \mathrm{M} \mathrm{KNO}_{3}\right)$ was titrated by successively adding $10 \mathrm{~mm} \mathrm{Ga}\left(\mathrm{NO}_{3}\right)_{3}$ at the molar ratio up to 200. The absorption and fluorescence spectra were recorded after about $10 \mathrm{~min}$ standing, at which time spectral changes were generally settled. The $\mathrm{pH}$ changes were also measured.

Spectrophotometric Titration of ADR-Ga(III) Complex An ADR $(40 \mu \mathrm{M})$ solution containing $4 \mathrm{~mm} \mathrm{Ga}\left(\mathrm{NO}_{3}\right)_{3}$ was titrated with a small amount of $0.02 \mathrm{M} \mathrm{HCl}$ or $0.02 \mathrm{M} \mathrm{KOH}$. The absorbance changes at $567 \mathrm{~nm}$ in the $\mathrm{pH}$ region from 2.3 to 3.9 (final pHs after titration) were monitored and a stability constant was derived according to the Hildebrand-Reilley method. ${ }^{14)}$

In the meantime, ADR $(40 \mu \mathrm{M})$ was titrated by $\mathrm{Ga}(\mathrm{III})$ at molar ratios up to 175 where the $\mathrm{pH}$ was always maintained at 3.0 by adding $0.02 \mathrm{M}$ $\mathrm{HCl}$ or $0.02 \mathrm{M} \mathrm{KOH}$. The absorptivity at $567 \mathrm{~nm}$ was monitored and the stability constant estimated according to the Benesi-Hildebrand method. ${ }^{15}$ )

\section{Results and Discussion}

Deprotonation Constants of ADR The interactions of the ADR with metal ions and other biologically important substances depend on its various prototropic forms. The protolytic equilibria of the drug in citrate and phosphate buffers and sulfuric acid solution were intensively investigated in this respect. ${ }^{12)}$ Before studying a $\mathrm{Ga}(\mathrm{III})-$ ADR system where gallium was used as $\mathrm{Ga}\left(\mathrm{NO}_{3}\right)_{3}$, deprotonation constants of ADR were determined by spectrophotometric titration in $0.1 \mathrm{M} \mathrm{KNO}_{3}$.

Figure 1 shows the individual stage of protolytic equilibria of ADR with the microscopic dissociation constants, $K_{\mathrm{CN}}$, $K_{\mathrm{CZ}}, K_{\mathrm{NA}}$ and $K_{\mathrm{ZA}}$. Defined similarly to previous literature, ${ }^{12)}$ the $K_{\mathrm{CN}}$ and $K_{\mathrm{NA}}$ correspond to the equilibria 


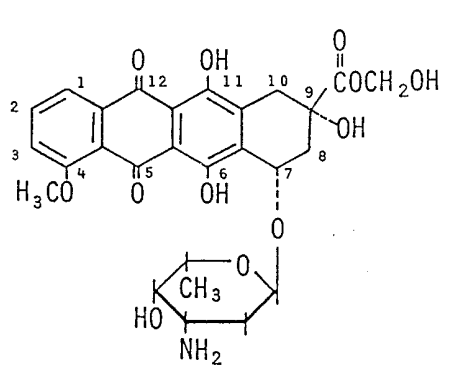

Fig. 1. Structure of Adriamycin and a Chart of Its Protolytic Equilibria

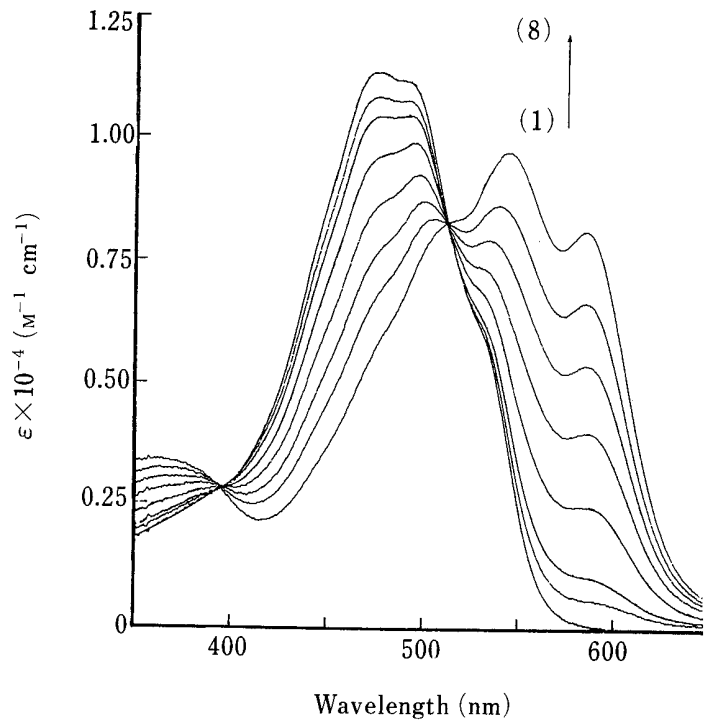

Fig. 2. Absorption Spectra of Adriamycin without $\mathrm{Ga}(\mathrm{III})$ as a Function of $\mathrm{pH}$ in $0.1 \mathrm{M} \mathrm{KNO}_{3}$

$\mathrm{pH}:(1), 6.39 ;(2), 8.33 ;(3), 8.79 ;(4), 9.26 ;(5), 9.57 ;(6), 9.82 ;(7), 10.06 ;(8), 10.34$

between the singly charged cation and the neutral species, and between neutral species and the singly charged anion, respectively. The $K_{\mathrm{CZ}}$ and $K_{\mathrm{ZA}}$ correspond to the equilibria between the singly charged cation and zwitterionic species, and between zwitterionic species and singly charged anion, respectively.

Figure 2 shows the visible absorption spectra of ADR in the $\mathrm{pH}$ range $6.39-10.34$. As the $\mathrm{pH}$ was raised, the absorbance at $480 \mathrm{~nm}$ decreased and the band shifted to a higher wavelength at maxima 555 and $590 \mathrm{~nm}$ with the isosbestic points at 398 and $517 \mathrm{~nm}$ (Fig. 2). The color of ADR solution changed from red to purple at basic $\mathrm{pH}$. This large shift can be attributed to the deprotonation of one phenolic group. ${ }^{4 b, d)}$ Since the amino group of the amino sugar moiety is located apart from the aromatic chromophore of the aglycone, the deprotonation of the amino group is unlikely to affect the spectrum of ADR.

The microscopic constants represented in Fig. 1 have the following relation to the macroscopic constants, $K_{1}$ and $K_{2}$, which represent first and second deprotonation, respectively. ${ }^{12)}$

$$
\begin{aligned}
& K_{1}=K_{\mathrm{CN}}+K_{\mathrm{CZ}} \\
& \frac{1}{K_{2}}=\frac{1}{K_{\mathrm{NA}}}+\frac{1}{K_{\mathrm{ZA}}}
\end{aligned}
$$
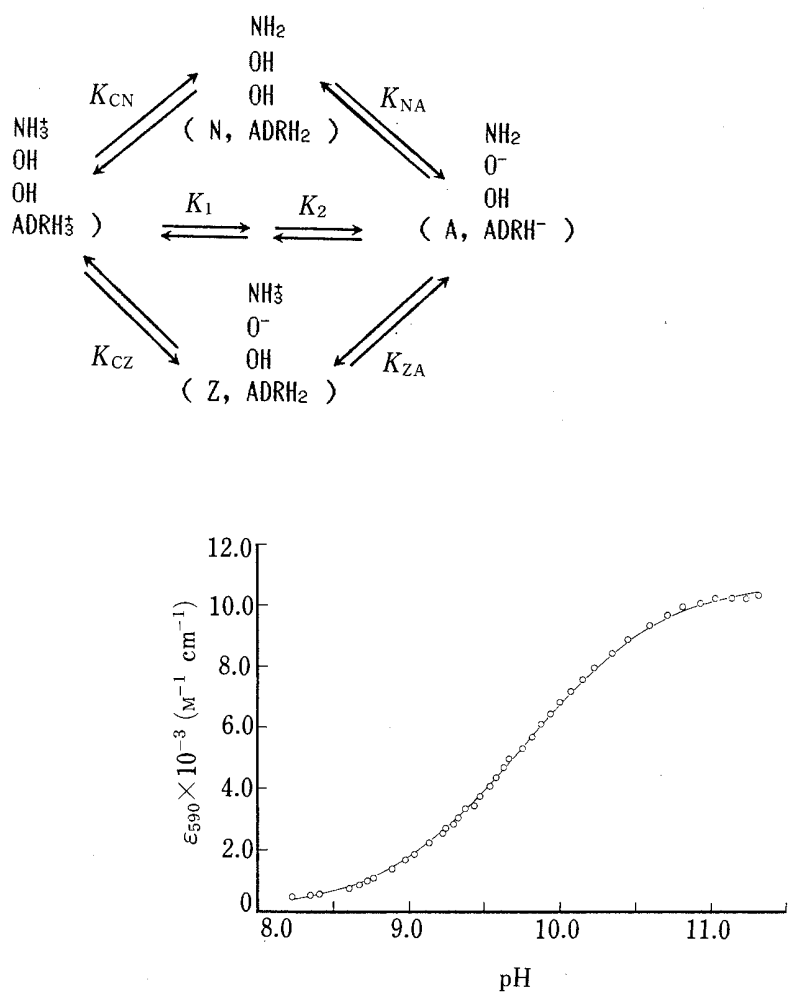

Fig. 3. Spectrophotometric Titration of Adriamycin at $590 \mathrm{~nm}$ in $0.1 \mathrm{MKNO}_{3}$

ADR concentration was $9.9 \times 10^{-6} \mathrm{M}$. Titration was carried out using $74.8 \mathrm{mM}$ $\mathrm{KOH}$. A solid line represents the calculated absorbance changes using the parameters obtained.

TABLE I. Negative Logarithms of Dissociation Constants of Adriamycin

\begin{tabular}{ll}
\hline Constant & Value \\
\hline $\mathrm{p} K_{1}$ & 9.10 \\
$\mathrm{p} K_{2}$ & 9.97 \\
$\mathrm{p} K_{\mathrm{CN}}$ & 9.25 \\
$\mathrm{p} K_{\mathrm{CZ}}$ & 9.64 \\
$\mathrm{p} K_{\mathrm{ZA}}$ & 9.43 \\
$\mathrm{p} K_{\mathrm{NA}}$ & 9.82 \\
\hline
\end{tabular}

The reproducibilities of the $K_{1}$ and $K_{2}$ values obtained from Eq. 3 by nonlinear least-squares analysis were within $9 \%$ and $7 \%$, respectively.

Based on these equilibria, the observed molar absorptivity of $\mathrm{ADR}, \varepsilon$, corresponding to total absorbance at an analytical wavelength may be expressed by

$$
\varepsilon=\frac{\varepsilon_{\mathrm{C}}\left[\mathrm{H}^{+}\right]^{2}+K_{1}\left[\mathrm{H}^{+}\right] \varepsilon_{\mathrm{N}^{\prime}}+K_{1} K_{2} \varepsilon_{\mathrm{A}}}{\left[\mathrm{H}^{+}\right]^{2}+K_{1}\left[\mathrm{H}^{+}\right]+K_{1} K_{2}}
$$

where $\varepsilon_{\mathrm{C}}[\mathrm{N}]+\varepsilon_{\mathrm{A}}[\mathrm{Z}]=\varepsilon_{\mathrm{N}^{\prime}}([\mathrm{N}]+[\mathrm{Z}])$, and $\varepsilon_{\mathrm{C}}, \varepsilon_{\mathrm{N}^{\prime}}$ and $\varepsilon_{\mathrm{A}}$ refer to the species $\mathrm{ADRH}_{3}^{+}, \mathrm{ADRH}_{2}$ and $\mathrm{ADRH}^{-}$, respectively. As the fully protonated species does not absorb at $590 \mathrm{~nm}\left(\varepsilon_{\mathrm{C}}=0\right)$, the absorptivity at $590 \mathrm{~nm}$ was used to determine deprotonation constants, $K_{1}$ and $K_{2}$. Figure 3 shows the plot of $\varepsilon_{590}$ as a function of $\mathrm{pH}$. When the value of $\varepsilon_{\mathrm{A}}, 10800$ was held constant, the parameters $\varepsilon_{\mathrm{N}^{\prime}}, K_{1}$ and $K_{2}$ were estimated using Eq. 3. The ratio of [N] to [Z], represented as $\left(\varepsilon_{\mathrm{A}}-\varepsilon_{\mathrm{N}^{\prime}}\right) /\left(\varepsilon_{\mathrm{N}^{\prime}}-\varepsilon_{\mathrm{C}}\right)$, was calculated to be 2.45 . Thus, according to Eqs. 1 and 2, the microscopic constants were obtained as summarized in Table I. A solid line in Fig. 3 represents the calculated curve based on these values.

Spectral Changes of Ga(III)-ADR Complex Formation 


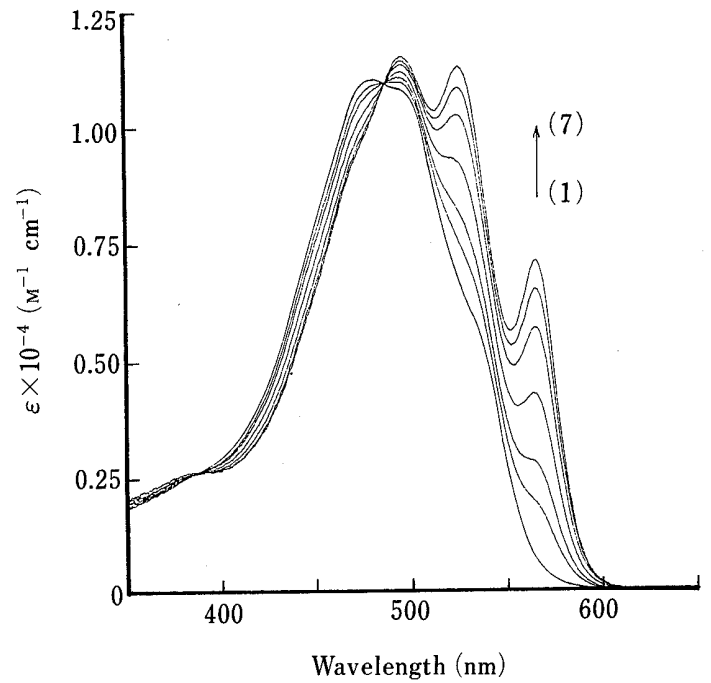

Fig. 4. Absorption Spectra of Adriamycin Titrated by Gallium Nitrate ADR concentration was $4.0 \times 10^{-5} \mathrm{M}$. [Ga( $\left.\left(\mathrm{NO}_{3}\right)_{3}\right] /[\mathrm{ADR}]$ and $\mathrm{pH}:(1), 0,6.46$; (2), 10, 3.43; (3), 20, 3.28; (4), 50, 3.11; (5), 100,3.00; (6), 150, 2.95; (7), 200, 2.91 in $0.1 \mathrm{M} \mathrm{KNO}_{3}$.

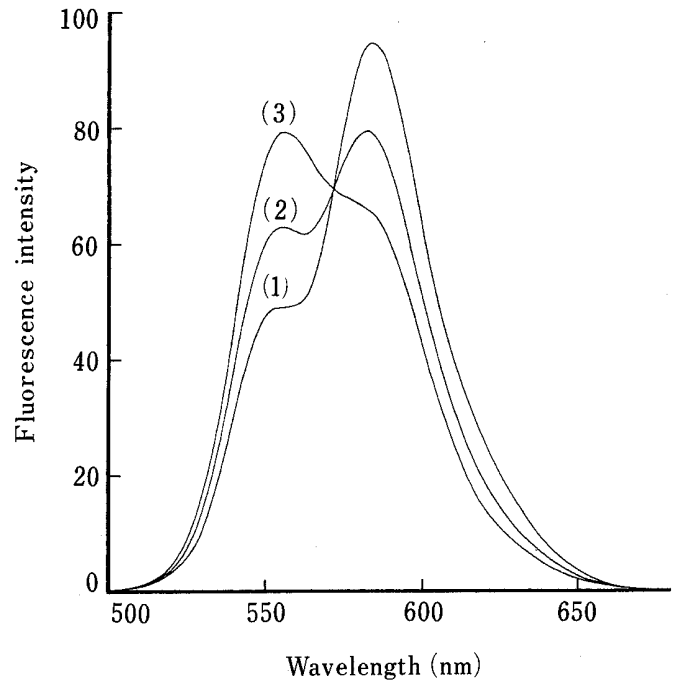

Fig. 5. Fluorescence Spectra of Adriamycin in the Presence of $\mathrm{Ga}\left(\mathrm{NO}_{3}\right)_{3}$ ADR concentration was $4.0 \times 10^{-5} \mathrm{M} .\left[\mathrm{Ga}\left(\mathrm{NO}_{3}\right)_{3}\right] /[\mathrm{ADR}]$ and $\mathrm{pH}$ : (1), 0, 6.46; (2), $50,3.11 ;(3), 100,3.00$ in $0.1 \mathrm{M} \mathrm{KNO}_{3}, \lambda_{\mathrm{ex}}=460 \mathrm{~nm}$.

Figure 4 shows the spectral changes of ADR in the presence of various amounts of $\mathrm{Ga}\left(\mathrm{NO}_{3}\right)_{3}$ in which the molar ratio of the metal ion to the drug varied from 0 to 200 . The scanning spectra exhibited new absorption maxima at 496,526 and $567 \mathrm{~nm}$, accompanied by color changes from red to red-purple. The spectral shift was more appreciable, especially when $\mathrm{Ga}$ (III) was more than 10 -fold the amount of ADR. As no spectral changes of the drug were observed in the acidic region, these shifts are the result of induction by $\mathrm{Ga}(\mathrm{III})$ and suggest the formation of a complex between $\mathrm{Ga}(\mathrm{III})$ and ADR. The spectra also exhibited isosbestic points at 387 and $487 \mathrm{~nm}$, showing that a single gallium-ADR complex is formed. We also performed a spectroscopic study at physiological $\mathrm{pH}$, but a shift of spectra was not detected. This may have been due to hydrolysis of gallium ion, which will be discussed later.

Concerning the complex formation, there are three possibilities: Ga(III) ion is bound to ADR through (i) the

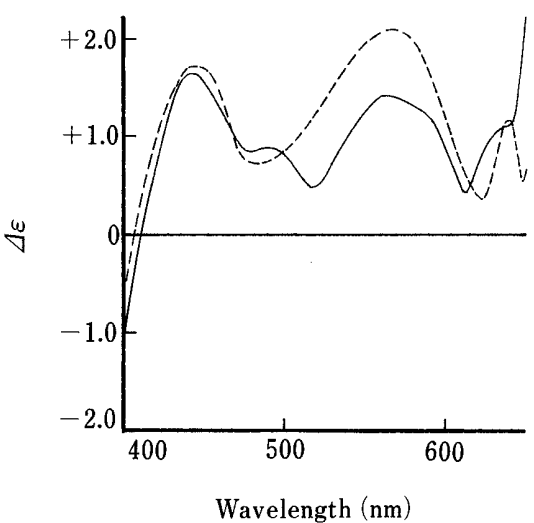

Fig. 6. CD Spectra of Adriamycin in the Absence and Presence of $\mathrm{Ga}\left(\mathrm{NO}_{3}\right)_{3}$ at $\mathrm{pH} 3.0$

ADR concentration was $4.0 \times 10^{-5} \mathrm{M} .\left[\mathrm{Ga}\left(\mathrm{NO}_{3}\right)_{3}\right]:---, 0 \mathrm{M} ;-, 6.0 \times 10^{-3} \mathrm{M}$ in $0.1 \mathrm{M} \mathrm{KNO}_{3}$.

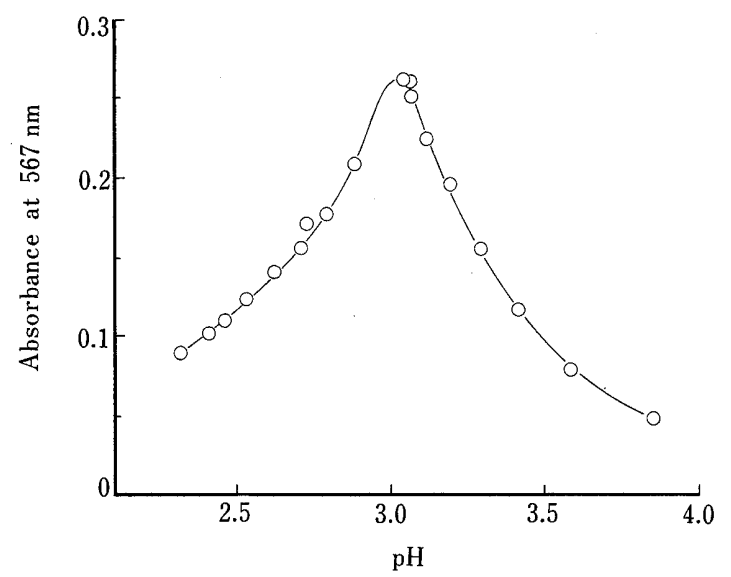

Fig. 7. Spectrophotometric Titration of $\mathrm{Ga}(\mathrm{III})$-Adriamycin Complex $[\mathrm{ADR}], 4.0 \times 10^{-5} \mathrm{M} ;\left[\mathrm{Ga}\left(\mathrm{NO}_{3}\right)_{3}\right], 4.0 \times 10^{-3} \mathrm{M}$. Titration was carried out using $0.02 \mathrm{M} \mathrm{KOH}$ or $\mathrm{HCl}$ in $0.1 \mathrm{M} \mathrm{KNO}_{3}$.

hydroxyl group on $\mathrm{C}_{11}$, (ii) the hydroxyl group on $\mathrm{C}_{6}$, or (iii) the amino group of the sugar. The shift of the band to higher wavelengths could be assigned to deprotonation of the phenolic groups. ${ }^{4 b, d)}$ Thus, the observation suggests that the first or the second is feasible.

Figure 5 shows representative fluorescence spectra of ADR in the presence of $\mathrm{Ga}\left(\mathrm{NO}_{3}\right)_{3}$ under similar conditions to Fig. 4. It exhibits an isoemissive point at $572 \mathrm{~nm}$. This also strongly suggests the involvement of phenolic chromophores in reference to the fluorescence spectrum of ADR at alkaline $\mathrm{pH}^{12)}$ Considering the similarity to the complexes formed between ADR and $\mathrm{Fe}(\mathrm{III}), \mathrm{Cu}(\mathrm{II})$, or $\mathrm{Pd}(\mathrm{II}),{ }^{3,4 b, 5)} \mathrm{Ga}(\mathrm{III})$ is most probably bound to the $\mathrm{C}_{11}$-phenolate oxygen and the $\mathrm{C}_{12}$-carbonyl oxygen of ADR.

It has been shown that the dimeric form of ADR could accommodate two Pd(II) ions. ${ }^{5)}$ In the case of the Ga(III)-ADR system, the existence of the dimer of ADR was examined by $C D$ spectrum. Figure 6 shows the $C D$ spectra at the molar ratios of $\mathrm{Ga}$ (III) to ADR $0: 1$ and $150: 1$ when the $\mathrm{pH}$ was maintained at 3.0. If ADR were in the dimeric form, the CD signal should be of the couplet type with a positive band at $445 \mathrm{~nm}$ and a negative one at $530 \mathrm{~nm}$, while if it were in the monomeric form, only one positive band at $480 \mathrm{~nm}$ should appear. ${ }^{16)}$ As the CD spectrum exhibited 
no negative band at $530 \mathrm{~nm}$ in the Ga(III)-ADR system, it can be ruled out that $\mathrm{Ga}$ (III) ions are bound to the dimeric form of ADR.

Stability Constants for Ga(III)-ADR Complex Formation Figure 7 shows spectrophotometric titration by the addition of $0.02 \mathrm{M} \mathrm{KOH}$ or $0.02 \mathrm{M} \mathrm{HCl}$ when the molar ratio of $\mathrm{Ga}(\mathrm{III})$ to ADR was held constant at 100 . The absorbance at $567 \mathrm{~nm}$ reflecting the complex formation was monitored in the $\mathrm{pH}$ region $2.3-3.8$. At $\mathrm{pHs}$ ranging from 2.3 to 3.0 the absorbance increased with increasing $\mathrm{pH}$ value as the result of complexation. The maximum absorbance was shown at $\mathrm{pH} 3.0$, followed by diminished absorbance with further increase in $\mathrm{pH}$. The decreased absorbance observed in the $\mathrm{pH}$ region greater than 3.0 could be explained by hydrolysis of the $\mathrm{Ga}(\mathrm{III})$ ion. The gallium ion is hydrolyzed to form $\mathrm{Ga}(\mathrm{OH})^{2+}, \mathrm{Ga}(\mathrm{OH})_{2}^{+}$and $\mathrm{Ga}(\mathrm{OH})_{3} \cdot{ }^{17,18)}$ The values of the hydrolysis constants of gallium have been reported previously, ${ }^{18)}$ with $\log K_{\mathrm{OH}}^{1}=2.91, \log K_{\mathrm{OH}}^{2}=3.70$ and $\log K_{\mathrm{OH}}^{3}=4.40$ corresponding to the formation of the species described above, respectively. The prevailing forms of the metal in the $\mathrm{pH}$ region examined $(2.3-3.8$, Fig. 7) are $\mathrm{Ga}^{3+}$ and $\mathrm{Ga}(\mathrm{OH})^{2+}$. Therefore, the concentration of the unhydrolyzed metal ion decreases with increasing $\mathrm{pH}$.

To estimate the stability constant of the complex, the following two methods were used. First, the $\mathrm{pH}$-absorption profile reflecting the complex formation shown in Fig. 7 was analyzed according to the method of Hildebrand and Reilley. ${ }^{14)}$ Assuming that the $\mathrm{Ga}^{3+}$ species is responsible for the complex formation and the complex is formed at a $1: 1$ stoichiometry, the stability constant, $\beta$, is defined as follows:

$$
\begin{aligned}
& \mathrm{Ga}^{3+}+\mathrm{ADRH}_{2} \rightleftharpoons\left[\mathrm{Ga}\left(\mathrm{ADRH}_{2}\right)\right]^{3+} \\
& \beta=\frac{\left[\mathrm{Ga}\left(\mathrm{ADRH}_{2}\right)^{3+}\right]}{\left[\mathrm{Ga}^{3+}\right]\left[\mathrm{ADRH}_{2}\right]} \\
& \mathrm{Ga}^{3+}+\mathrm{ADRH}_{3}^{+} \rightleftharpoons\left[\mathrm{Ga}\left(\mathrm{ADRH}_{2}\right)\right]^{3+}+\mathrm{H}^{+} \\
& \beta^{*}=\frac{\left[\mathrm{Ga}\left(\mathrm{ADRH}_{2}\right)^{3+}\right]\left[\mathrm{H}^{+}\right]}{\left[\mathrm{Ga}^{3+}\right]\left[\mathrm{ADRH}_{3}^{+}\right]}
\end{aligned}
$$

where $\beta^{*}$ is the equilibrium constant. The logarithmic form of Eq. 5 is given by

$$
\log \frac{\left[\mathrm{Ga}\left(\mathrm{ADRH}_{2}\right)^{3+}\right]}{\left[\mathrm{ADRH}_{3}^{+}\right]}-\log \left[\mathrm{Ga}^{3+}\right]=\mathrm{pH}-\mathrm{p} \beta^{*}
$$

in which $\mathrm{p} \beta^{*}$ means $\left(-\log \beta^{*}\right)$. The first term of the left member can be represented by

$$
\log \frac{\left[\mathrm{Ga}\left(\mathrm{ADRH}_{2}\right)^{3+}\right]}{\left[\mathrm{ADRH}_{3}^{+}\right]}=\log \frac{A-A_{\mathrm{f}}}{A_{\infty}-A}
$$

where $A$ is absorbance for ADR in the presence of excess $\mathrm{Ga}$ (III) concentrations, $A_{\mathrm{f}}$ the absorbance for free ADR which is constant in the acidic region, and $A$ the absorbance when ADR is fully complexed with $\mathrm{Ga}(\mathrm{III})$. On plotting the absorbance changes according to Eqs. 6 and 7, it should be born in mind that the concentration of unhydrolyzed metal species, $\left[\mathrm{Ga}^{3+}\right]$, in Eq. 6 could not always be approximated to the initial concentration of the metal ion if the metal ion were in excess of the drug. As mentioned earlier, this is because the concentration of the $\mathrm{Ga}^{3+}$ species is determined by the hydrolysis constants and $\mathrm{pH}$ examined. A complex formation may also diminish the unhydrolyzed metal ion. However, the influence of the $\mathrm{pH}$ changes is likely to be much larger than that of the complex formation because of an excess metal ion compared to the drug. Therefore, the analysis of the spectral changes was made in the lower $\mathrm{pH}$ region $(2.28-2.62)$ to avoid such complexity where the effective concentration of the $\mathrm{Ga}^{3+}$ species was adjusted by only the hydrolysis constant at each $\mathrm{pH}$ examined.

Figure 8 shows the relationship between the absorbance changes reflecting the complex formation monitored at $567 \mathrm{~nm}$ and the $\mathrm{pH}$ according to Eqs. 6 and 7. The plot showed a linear dependency on the $\mathrm{pH}$, producing a slope of 1.21. This result supports the idea that one proton is most likely to be released from the ADR as a result of the $1: 1$ complex formation. Subsequently, the equilibrium constant $\beta^{*}$ could be estimated from the intercept on the ordinate according to Eq. 6. Taking in the first deprotonation constant, $K_{1}$ and the $\beta^{*}$ value obtained, $9.82 \times$ $10^{-2}$, the stability constant $\left(\beta=\beta^{*} / K_{1}\right)$ thus obtained was given as $1.24 \times 10^{8} \mathrm{M}^{-1}$.

Secondly, the stability constant was determined by the method of Benesi-Hildebrand. ${ }^{15)}$ In a set of experiments in which ADR $(40 \mu \mathrm{M})$ was titrated by $\mathrm{Ga}\left(\mathrm{NO}_{3}\right)_{3}$ at molar ratios up to 175 , the $\mathrm{pH}$ was held constant at 3.0 by adding

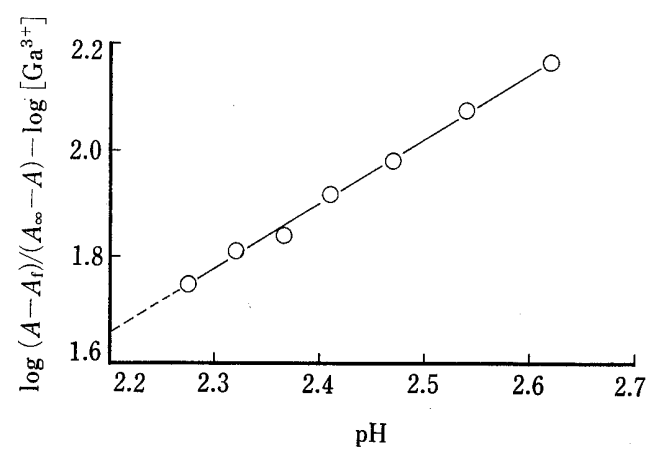

Fig. 8. Plots for Determining the Equilibrium Constant $\left(\beta^{*}\right)$ of Ga(III)-Adriamycin Complex According to Eqs. 7 and 8

The effective concentration of $\mathrm{Ga}$ (III) was adjusted by the hydrolysis constant of the metal ion with changes of $\mathrm{pH}$. Temperature: $25^{\circ} \mathrm{C}$.

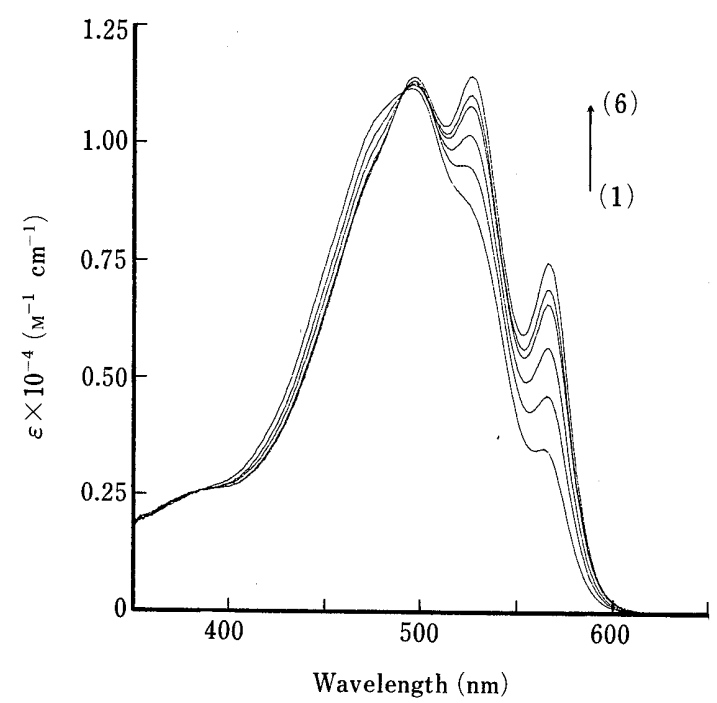

Fig. 9. Absorption Spectra of $\mathrm{Ga}$ (III)-Adriamycin Solution at $\mathrm{pH} 3.0$ ADR concentration was $4.0 \times 10^{-5} \mathrm{M}$. [Ga( $\left.\left.\mathrm{NO}_{3}\right)_{3}\right] /[\mathrm{ADR}]:(1), 50 ;(2), 75 ;$ (3), 100; (4), 125; (5), 150; (6), 175 in $0.1 \mathrm{M} \mathrm{KNO}_{3}$. 


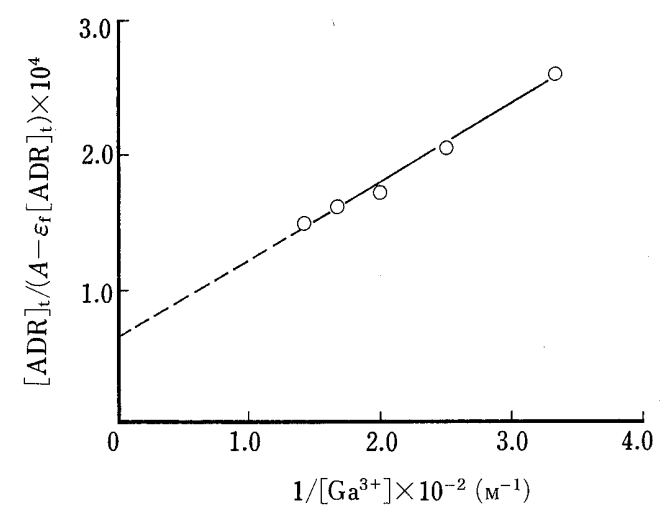

Fig. 10. Double-Reciprocal Plots for Determining the Stability Constant $(\beta)$ of $\mathrm{Ga}$ (III)-Adriamycin Complex According to Eq. 9

Temperature: $25^{\circ} \mathrm{C}$.

$0.02 \mathrm{M} \mathrm{HCl}$ or $0.02 \mathrm{M} \mathrm{KOH}$. Figure 9 shows spectral changes as a function of the metal concentration. Assuming that the $1: 1$ complex formation is responsible for these changes, the following equilibrium equations can be derived:

$$
\begin{aligned}
& \mathrm{Ga}^{3+}+\mathrm{ADRH}_{3}^{+} \rightleftharpoons\left[\mathrm{Ga}\left(\mathrm{ADRH}_{2}\right)\right]^{3+}+\mathrm{H}^{+} \\
& K=\beta^{*} /\left[\mathrm{H}^{+}\right]=\frac{\left[\mathrm{Ga}\left(\mathrm{ADRH}_{2}\right)^{3+}\right]}{\left[\mathrm{Ga}^{3+}\right]\left[\mathrm{ADRH}_{3}^{+}\right]} \\
& \frac{[\mathrm{ADR}]_{\mathrm{t}}}{A-\varepsilon_{\mathrm{f}}[\mathrm{ADR}]_{\mathrm{t}}}=\frac{1}{K\left(\varepsilon_{\mathrm{b}}-\varepsilon_{\mathrm{f}}\right)} \cdot \frac{1}{\left[\mathrm{Ga}^{3+}\right]}+\frac{1}{\varepsilon_{\mathrm{b}}-\varepsilon_{\mathrm{f}}}
\end{aligned}
$$

where $K$ is an arbitrary constant. $A$ is the absorbance at $567 \mathrm{~nm} . \varepsilon_{\mathrm{f}}$ and $\varepsilon_{\mathrm{b}}$ represent molar absorptivities for free ADR and complexed ADR, respectively. [ADR $]_{t}$ is the initial concentration of ADR. $\left[\mathrm{Ga}^{3+}\right]$ is the concentration of the unhydrolyzed metal ion calculated from the initial concentration and the hydrolysis constant at $\mathrm{pH}$ 3.0.

Figure 10 shows a plot according to Eq. 9, which allowed calculation of the $K$ value, $2.43 \times 10^{2} \mathrm{M}^{-1}$, from the slope and subsequently the stability constant. The stability constant $\left(\beta=K\left[\mathrm{H}^{+}\right] / K_{1}\right) 3.06 \times 10^{8} \mathrm{M}^{-1}$, finally obtained was in good agreement with that obtained by the previous method.

The complex formation between ADR and Ga(III) was only observed in the low $\mathrm{pH}$ region. It is therefore most probable that ADR is unable to form complexes with $\mathrm{Ga}(\mathrm{III})$ as the $\mathrm{pH}$ increases because of the hydrolysis of the metal ion. In contrast with the above, such a phenomenon was not found in the complexation of $\mathrm{ADR}-\mathrm{Fe}$ (III), in which the spectroscopic titration data showed an absorbance plateau with increasing $\mathrm{pH}$ up to 7 , although
Fe(III) also formed ferric hydroxide. ${ }^{3)}$ Precise kinetic data including the hydration rate of the metal ions may be needed to explain this difference. The intrinsic difference of these metal ions is that $\mathrm{Ga}$ (III) is a trivalent $\mathrm{d}^{10}$-system metal ion while $\mathrm{Fe}$ (III) belongs to transition elements, possibly making use of its $3 d, 4 s$ and $4 p$ orbitals in forming hybrids. Finally, the lack of complex formation of ADR and Ga(III) in the neutral $\mathrm{pH}$ region seems to be at a disadvantage for therapeutic use because the complex would not be able to remain unmodified in biological fluids.

Acknowledgment The authors wish to acknowledge Dr. J. Hirose for helpful discussion and Mr. S. Ito for technical assistance. This research was supported in part by a Grant-in-Aid provided from the Ministry of Education, Science, and Culture of Japan.

\section{References and Notes}

1) F. Arcamone, "Metabolism of Xenobiotics," ed. by J. W. Gorrod, H. Oelschläger, and J. Caldwell, Taylor \& Francis, London, 1988, pp. $115-119$.

2) M. Gosalvez, M. F. Blanco, C. Vivero, and F. Valles, Eur. J. Cancer, 14, 1185 (1978); C. E. Myers, L. Gianni, C. B. Simone, R. Klecker and R. Greene, Biochemistry, 21, 1707 (1982).

3) H. Beraldo, A. Garnier-Suillerot, L. Tosi, and F. Lavelle, Biochemistry, 24, 284 (1985).

4) a) P. M. May, G. K. Williams, and D. R. Williams, Inorg. Chim. Acta, 46, 221 (1980); b) F. T. Greenaway and J. V. Dabrowiak, J. Inorg. Biochem., 16, 91 (1982); c) R. Kiraly and R. B. Martin, Inorg. Chim. Acta, 67, 13 (1982); d) H. Beraldo, A. Garnier-Suillerot, and L. Tosi, Inorg. Chem., 22, 4117 (1983).

5) M. M. L. Fiallo and A. Garnier-Suillerot, Biochemistry, 25, 924 (1986).

6) C. L. Edwards and R. L. Hayes, J. Nucl. Med., 10, 103 (1969).

7) E. Aulbert, A. Gibhardt, D. Shulz, and U. Hanbold, Nucl. Med., (Amsterdam) 15, 183 (1976); P. Bickel and H. A. Hansen, Br. J. Radiol., 45, 182 (1972).

8) H. Onishi, "Treatise on Analytical Chemistry," Part II, Vol. 2, ed. by I. M. Kolthoff and P. J. Elving, Interscience Publishers, Inc., New York, 1962, pp. 1-105.

9) J. B. Chaires, N. Dattagupta, and D. M. Crothers, Biochemistry, 21, 3927 (1982).

10) A. Albert and E. P. Serjeant, "Ionization Constants of Acids and Bases," Methuen and Co., Ltd., Great Britain, 1962.

11) J. H. Beijnen, O. A. G. J. van der Houwen, and W. J. M. Underberg, Int. J. Pharmaceut., 32, 123 (1986).

12) R. J. Sturgeon and S. G. Schulman, J. Pharm. Sci., 66, 958 (1977).

13) K. Yamaoka, Y. Tanigawara, T. Nakagawa, and T. Uno, $J$. Pharmacobio-Dyn., 4, 879 (1981).

14) G. P. Hildebrand and C. N. Reilley, Anal. Chem., 29, 258 (1957).

15) H. A. Benesi and J. H. Hildebrand, J. Am. Chem. Soc., 71, 2703 (1949).

16) S. R. Martin, Biopolymers, 19, 713 (1980).

17) W. R. Harris and A. E. Martell, Inorg. Chem., 15, 713 (1976).

18) V. A. Nazarenko, V. P. Antonovich, and E. M. Nevskaya, Russ. J. Inorg. Chem. (Engl. Transl.), 13, 825 (1968). 